Research Article

\title{
Development of a Hybrid Method to Predict the Slope Surface Deformation Utilizing the Time Series and GA-Elman Models
}

\author{
Gang Wang $\mathbb{D},,^{1,2}$ Zheng Fang $\mathbb{D}^{1},{ }^{1}$ Jiren Xie $\mathbb{D}^{1},{ }^{1}$ and $\mathrm{Na} \mathrm{Du} \mathbb{D D}^{3}$ \\ ${ }^{1}$ Department of Civil Engineering, Central South University, Changsha 410075, China \\ ${ }^{2}$ Department of Civil and Environmental Engineering, Tokyo Institute of Technology, Tokyo, Japan \\ ${ }^{3}$ School of Information and Communication Engineering, University of Electronic Science and Technology of China, \\ Chengdu 611731, China \\ Correspondence should be addressed to Zheng Fang; fangzheng2@csu.edu.cn
}

Received 26 October 2020; Revised 27 November 2020; Accepted 20 December 2020; Published 8 January 2021

Academic Editor: Chong Xu

Copyright (C) 2021 Gang Wang et al. This is an open access article distributed under the Creative Commons Attribution License, which permits unrestricted use, distribution, and reproduction in any medium, provided the original work is properly cited.

\begin{abstract}
A reliable prediction of the surface deformation of slopes is vital to better assess the fatalities and economic losses caused by landslides. Many prediction methods have been proposed to estimate the surface deformation of landslides with nonlinear characteristics. However, these methods have low accuracy and poor applicability. In this paper, a new hybrid method for surface deformation prediction was proposed, which was deduced from the Wavelet Analysis, Genetic Algorithm (GA), and Elman Algorithm. In this method, the slope surface deformation was decomposed into a trend component and a periodic component using the time series model, which were trained and predicted utilizing the GA-Elman model. The predicted slope surface deformation was the combination of the trend component and the periodic component. Then, the predicted results of slope surface deformation through GA-Elman were compared with the predicted results through Support Vector Machines (SVM), Extreme Learning Machine (ELM), Back Propagation (BP), and Genetic Algorithm-Back Propagation (GA-BP) models. The comparison was made with reference to the data retrieved from the on-site slopes and the laboratory tests. The results revealed that the proposed method highlighted reliability and could be used with higher accuracy to forecast the slope surface deformation in the process of landslides.
\end{abstract}

\section{Introduction}

Landslide is a natural disaster, which has posed a huge threat to people's lives and property. At the same time, it could also cause damage to the resource, the environment, and ecology, especially in remote mountainous and hilly areas [1-3]. Previous studies have shown that the slope surface deformation is a complicated multidimensional nonlinear dynamic system, which is affected by both internal and external factors $[4,5]$. As the internal development is affected by various conditions and the external interference factors are random, the prediction of the landslide is uncertain.

The prediction of the slope surface deformation has become one of the fundamental studies in slope disaster prevention [6]. The employment of the on-site monitoring data, such as inclination angle, displacement, water content, and pore water pressure, has a higher prediction accuracy $[7,8]$. There are mainly three kinds of models predicting the variations of slope surface deformation. One kind of prediction models is based on the on-site data and laboratory test results, among which the empirical model proposed by Saito is most widely used [9-11]. However, this model is not suitable for the prediction of the stepped and periodic landslides. Many researchers have established a series of statistical analysis models considering the slope surface characteristics and the time, which included the Verhulst model [12], Gray model [13], and Pearl Growth model [14]. However, such prediction models are case-specific, i.e., applicable to only one type of slope.

Recently, a large number of neural network prediction models, such as BP, ELM, Elman, SVM, and Wavelet Neural Network (WNN), have been employed to predict the slope 
surface deformations, which were also optimized through Genetic Algorithm, Artificial Bee Colony Algorithm, Chaotic Sequence Model, and Wavelet Analysis. Du et al. (2009) used the BP Neural Network to predict periodic component displacement. As there are too many parameters in the existing models, the prediction results are heavily affected by these parameters [15]. Zhang et al. (2015) adopted the polynomial and PSO-SVR to predict the slope surface displacement. However, the proposed fitting equation cannot reflect the internal factors of the slope [16]. Liu used SVM and Gaussian Process (GP) to simulate the slope displacement, which validated the optimization of the Gaussian Process [17]. Some difficulties still exist in the application of neural networks on the slope surface displacement prediction, such as weak generalization ability, considering many parameters, and so on. And the internal and external factors have not been distinguished in some research. Besides, most of the existing models focused on the prediction of the slope surface displacement without considering the effects of the inclination angle. The inclination angle can also reflect the evolution of the slope, which has a linear relation with the displacement $[18,19]$.

The Wavelet Analysis was employed to deal with the stepped variation trend of the slope surface displacement in this paper. The cumulative displacement is decomposed into trend component displacement and periodic component displacement by the time series model, after which the model adapted to the prediction of the slope surface displacement and inclination angle was established based on the Genetic Algorithm optimized Elman Neural Network. The proposed model was validated by the displacement data from the Baishuihe landslide in the Three Gorges areas and the inclination angle data from the laboratory tests.

\section{The Method of Predicting the Slope Surface Deformation}

The slope displacement or inclination angle was firstly denoised and normalized to obtain the typical slope surface deformation data. These data can be decomposed into a lowfrequency curve and several high-frequency curves through the discrete wavelet. The deformation of the periodic component and the trend component can be obtained through the combination of the high-frequency curves and the low-frequency curve. Approximately one-third of the original data is regarded as a simulation set, and the remaining data are regarded as a training set. Then, the optimized Elman Neural Network (Elman NN) based on the Genetic Algorithm is used to train and predict the slope deformation. Compared with the on-site monitoring date, the optimized model was validated. The flowchart of the data processing is shown in Figure 1.

2.1. Time Series Model. A nonlinear relationship was found between the slope displacement and the time (duration of landslides). However, some research showed that the inclination angle during sloping had a linear relation with the time. The slope deformation can be decomposed into the deformation of the trend component, the periodic component, and the random component [20], as follows:

$$
X(t)=m(t)+n(t)+\theta(t),
$$

where $X(t)$ is the surface deformation monitoring value; $m$ $(t)$ is the trend component surface deformation; $n(t)$ is the periodic component surface deformation; $\theta(t)$ is the random component surface deformation. As the effect of the random factor is negligible, it is not considered in this study [21]. Given the advantages of the Wavelet Analysis, the trend component deformation data are extracted, which will be discussed in detail in the following sections.

2.2. Discrete Wavelet Analysis. Wavelet Analysis can effectively identify the primary frequency components and then extract the local information, which can indicate the periodic variations hidden in time series [22]. The slope surface deformation data are generally discrete, which can be expressed as the function $f(k \Delta t)$. To the specific finite energy signal or integrable function $f(k \Delta t) \in L^{2} R$, the function can be transformed as follows:

$$
W_{f}(a, b)=|a|^{-(1 / 2)} \Delta t f(k \Delta t) \bar{\psi}\left(k \Delta t-\left(\frac{b}{a}\right)\right),
$$

where $W_{f}(a, b)$ is the coefficient of the wavelet transformation; $\psi(t)$ is the basis wavelet function; a is the scale factor; $b$ is the translation factor; $\bar{\psi}(k \Delta t-(b / a)$ is the complex conjugate function. Many researchers used the Mallat Algorithm in the discrete Wavelet Analysis [23], based on which the normalized slope deformation can be decomposed into a low-frequency component and several high-frequency components, as shown below:

$$
x_{i}=x_{l, i}+x_{h 1, i}+x_{h 2, i}+\cdots+x_{h L, i},
$$

where $L$ is the decomposition layer, $x_{l, i}$ is the low-frequency part after decomposition, which mainly represents the trend component deformation in the slope surface deformation; $x_{h 1, i}, x_{h 2, i}, x_{h L, i}$ is the first layer, the second layer, and the Lth layer in the high-frequency parts, indicating the periodic characteristics in the slope surface deformation.

The Daubechies wavelet $(\mathrm{dbN})$ has good positioning ability in the time domain and the frequency, which plays an important role in the decomposition of the discrete signals [24]. The db4 wavelet function, which is flat and smooth, is selected to decompose the signal in this study.

2.3. Optimization of the GA-Elman Model. A context layer is incorporated in the Elman Neural Network to achieve the memorization property. As a typical feedback type of the neural networks, the Elman Neural Network can make the system have the ability to adapt to the time-varying characteristics. The Elman Neural Network can reflect the learning process dynamically, which is suitable for the prediction of the time series. The network structure is shown in Figure 2.

There are $m$ nodes in the input layers, $n$ nodes in the output layers, and a context layers in the Elman network. 


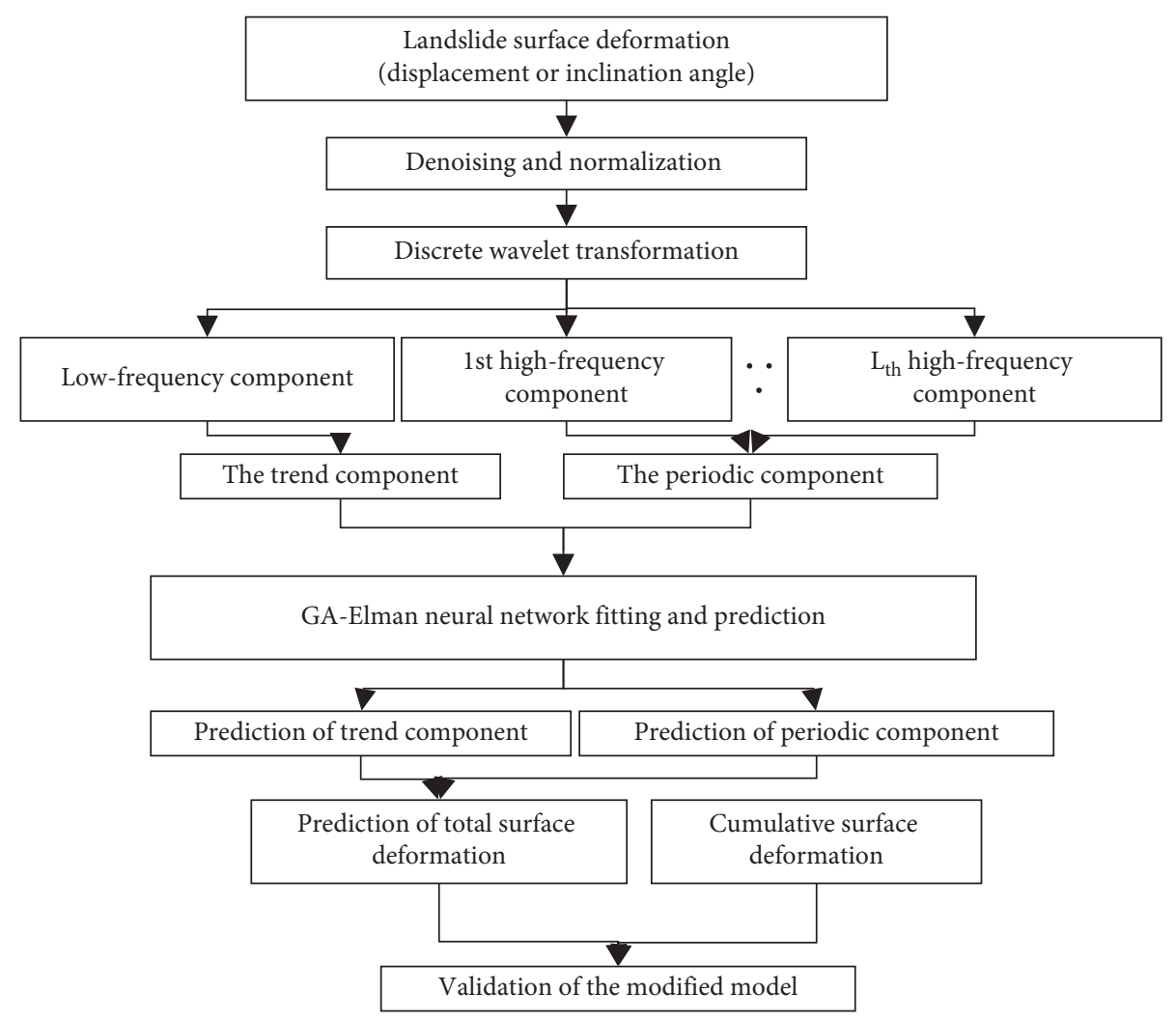

FIGURE 1: Flowchart of the WA-GA-Elman model (L denotes the number of the layer).



Figure 2: Topology structure of Elman neural network.

The connection weights between the input layer and the hidden layer, the hidden layer and the context layer, and the hidden layer and the output layer are $w 1, w 2$, and $w 3$, respectively. The input of the network is denoted by $i(k-1)$, the output of the hidden layer is represented by $x(k)$, the output of the context layer is represented by $x_{c}(k)$, and the output of the whole network is $y(x)$. Then, the following relations can be obtained:

$$
x(k)=f\left(w_{2} x_{c}(k)+w_{1}(i(k-1))\right),
$$

where

$$
x_{c}(k)=x(k-1),
$$

$f$ represents the transfer function, which is regarded as the sigmoid function; the equation is as follows: 


$$
f(x)=\left(1+e^{-x}\right)^{-1}
$$

The output of the neural network can be derived as follows:

$$
y(x)=g\left(w_{3} x(k)\right),
$$

where $g$ is the transfer function of the output layer, which is a linear function, whose connection weight is the same as that in the BP Neural Network. Therefore, the error of the Elman Neural Network can be obtained as follows:

$$
E=\sum_{k=1}^{m}\left(t_{k}-y_{k}\right)^{2}
$$

where $t_{k}$ is the output vector.

The Elman Neural Network has spatial memory capabilities and lag effects, which also inherits some shortcomings of the BP neural work, such as the failure of training and overfitting. The Genetic Algorithm can solve these problems. During the optimization of the connection weight and the threshold value of the neural network, the type of error function is not considered. Some penalty terms are inserted into the error function, which can improve the versatility and complexity of the network regardless of whether the error function is differentiable or not. This has great potential in the improvement of weight optimization.

During the construction of the GA-Elman algorithm, the main things are encoding the chromosome, defining the adaptability function, and constructing the genetic operators. Figure 3 shows the optimized algorithm structure of the GA-Elman model. The optimization focuses on accelerating the convergence and reducing training time through the determination of the connection weights and the threshold values, which can improve the operating efficiency of the neural network and predict the slope surface deformation accurately.

In the process of establishing the neural network, the parameters in the GA-Elman Neural Network are preset.

2.3.1. Groups Encoding. The individual in the groups is coded by binary, which means that the individual is a single binary string. The coding length can be represented as follows:

$$
L=(m \times h+h+h \times h+h \times n+n) \times M,
$$

where $L$ is the length of encoding; $m$, h, $n$ represent the nodes of the input layer, the hidden layer, and the output layer, respectively. $M$ is the bit number of the threshold and the weight value in the binary coding.

2.3.2. Adaptability Function. The adaptability function is allocated in the sorted method, as follows:

$$
\text { FitnV = ranking (obj), }
$$

where obj is the output of the operated objective function. Ranking() is the ranking function, FitnV is the adaptability function.
2.3.3. Constructing Genetic Operators. Genetic operators include selection operators and mutation operators. The selection of the selection operators depends on the random traversal sampling. The crossover operator selects the singlepoint crossover operator. The mutation operator can determine the number of mutated genes through the same probability. The mutated genes can be selected, and then the value can converse.

2.4. Accuracy Evaluation. The evaluation of the accuracy of the fitting and prediction is expressed by three indicators: Mean Absolute Percentage Error (MAPE), Root Mean Square Error (RMSE), and goodness of fit (R2). RMSE and R2 are used to describe the accuracy of the fitting process, while RMSE and MAPE are employed to describe the accuracy of the data prediction.

When the prediction value and the true value are the same, RMSE equals 0 . The greater the error, the greater the value. The equation of the RMSE is as below:

$$
\mathrm{RMSE}=\sqrt{\sum_{i=1}^{N_{0}}\left(\frac{\left(x_{\mathrm{old}, i}-\hat{y}_{i}\right)^{2}}{N_{0}}\right)},
$$

where $x_{\mathrm{old}, i}$ is the monitoring slope surface deformation data; $\widehat{y}_{i}$ is the fitted or the predicted slope surface deformation data; $N_{0}$ is the number of the fitting or predicted cases. To evaluate the fitting behavior of the model better, R2 is introduced, which ranges from 0 to 1 . The equation is as follows:

$$
\begin{aligned}
R^{2} & =\frac{\left(1-\sum_{i=1}^{N_{0}}\left(\hat{y}-x_{\mathrm{old}, i}\right)^{2}\right)}{\sum_{i=1}^{N_{0}}\left(\hat{y}-x_{\mathrm{old}, i}\right)^{2}}, \\
\text { MAPE } & =\frac{1}{N_{0}} \sum_{i=1}^{N_{0}}\left|\left(\frac{\left(x_{\mathrm{old}, i}-\hat{y}_{i}\right)}{x_{\mathrm{old}, i}}\right)\right| .
\end{aligned}
$$

The MAPE is the average absolute percentage error, which is usually used to predict the performance of evaluation indicators. When the MAPE equals 0 , the model can predict the real situation perfectly. The greater the MAPE, the greater deviation from the prediction mode.

\section{Prediction on the Slope Displacement of the Baishuihe}

Frequent landslides occurred in the Three Gorges area in China due to the special geographical and complex climatic conditions and frequent water level changes. By the year 2014, more than 5000 landslide points occurred in the Three Gorges area [25]. The study on the slope surface deformation and the prediction of the sliding laws is an important way to reduce and prevent landslide disasters [26].

3.1. Overview of the Baishuihe Landslide. The Baishuihe landslide is located on the south bank of the Yangtze River in Zigui County, the Three Gorges Reservoir area, which is 


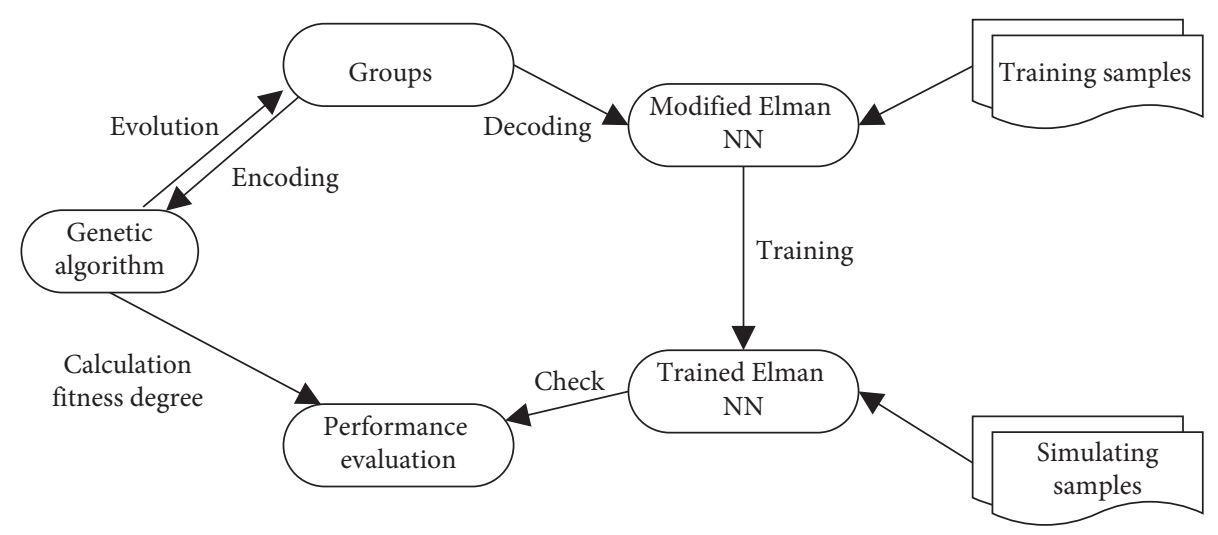

FIgURE 3: Flow chart of GA-Elman algorithm.

$56 \mathrm{~km}$ from the Three Gorges Dam site. Many bedding landslides have occurred in the past, which belonged to the loose accumulation landslides [27]. The slope is located in the wide valley area of the Yangtze River, which is a onesided oblique, step-shaped, high in the south, and low in the north. The length in the east-west direction is $700 \mathrm{~m}$, and the length in the north-south direction is $600 \mathrm{~m}$. The average thickness of the entire slope is $30 \mathrm{~m}$, and the total volume is $1260 \times 104 \mathrm{~m} 3$, as shown in Figure 4 . There are 9 GPS deformation monitoring points setting on the slope surface.

The recorded data of the ZG93 displacement monitoring point from June 2003 to March 2013 is selected, whose cumulative displacement curve is plotted in Figure 5. The cumulative displacement is decomposed into trend component displacement and the periodic component displacement by the Matlab Wavelet Analysis toolbox [28]. Then, the modified GA-Elman model is employed for fitting and prediction analysis.

3.2. Displacement Decomposition of the ZG93 Slope Displacement Time Series. The slope displacement sequence is decomposed into the low-frequency sequence and the highfrequency sequence through the $\mathrm{db} 4$ wavelet function. After several calculations, the cumulative displacement of the ZG93 monitoring point from 2003 to 2013 is decomposed into a low-frequency trend term and 5 high-frequency curves. The equation is as follows:

$$
x_{i}^{\mathrm{ZG} 93}=x_{l 1}^{\mathrm{ZG} 93}+x_{h 1}^{\mathrm{ZG} 93}+x_{h 2}^{\mathrm{ZG} 93}+x_{h 3}^{\mathrm{ZG} 93}+x_{h 4}^{\mathrm{ZG} 93}+x_{h 5}^{\mathrm{ZG} 93} .
$$

After extracting the trend component cumulative displacement curve (low-frequency curve) and recombining the high-frequency curves, the cumulative displacement periodic component curve of the Baishuihe can be obtained. The trend component displacement curve is smooth and complete, which is also without the obvious inflection points (Figure 6(a)). It can reflect the general trend of the displacement, and the periodic component displacement can show the obvious periodicity (Figure 6(b)).
3.3. Fitting and Prediction of the Trend Component Displacement. The proposed GA-Elman Neural Network is used to fit and predict the trend and component term. Some model parameters in the above prediction models need to be optimized through training (i.e., the kernel type and penalty parameter $c$ in the SVM, the number of nodes in hidden layers of $\mathrm{BP}$, the population size, the population size, and so on in GA-Elman network). After obtaining the optimized model parameters, the models are employed to predict the test data. The accuracy of the fitting and prediction results can be assessed through the accuracy evaluation indicators mentioned above. In this case, the data from July 2003 to June 2011 are imported into the GA-Elman for training/ fitting. After confirming the applicability of the models, we can obtain the prediction data from July 2011 to March 2013 through the same models. Compared with the monitoring data, the predictability of the models can be evaluated.

The main input control parameters in the Genetic Algorithm include the population size (80), the crossover probability (0.7), and the mutation probability (0.01) [29]. When there are 20 nodes in the hidden layers in the structure of the Elman Neural Network and the maximum number of iterations is set as 5000, the model has a good fitting effect. The fitting curves are shown in Figure 7 . The fitting curve agrees well with the trend component displacement. The R2 is 1 , and the RMSE is 2.2347 . The prediction displacement curve is shown in Figure 8(a), and it is in good agreement with the monitoring data recorded around 20 months. The RMSE is 3.9101, and the MAPE is 0.1643 , which indicates that the model can be used to predict the slope displacement.

To show the superiority of the GA-Elman Neural Network algorithm based on the WA, the fitting and prediction curves obtained from some static neural network models (BP, GABP, ELM, SVM) and the Elman network that has not been optimized by the Genetic Algorithm are compared. The RBF kernel function is used in the SVM Neural Network [30]; when the hidden layer nodes are 30, the ELM can perform better prediction behavior with the use of the sigmoid function [31]. The prediction curves based on different models are shown in Figure 8. The evaluation values of the different models are calculated and summarised in Table 1. 


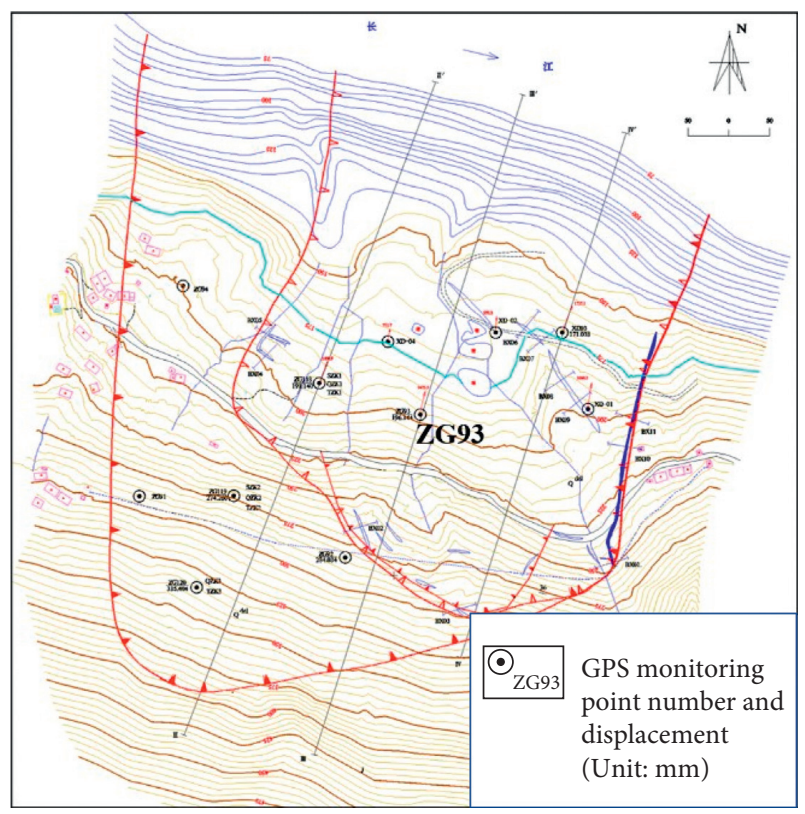

Figure 4: 2D map of Baishuihe landslide.

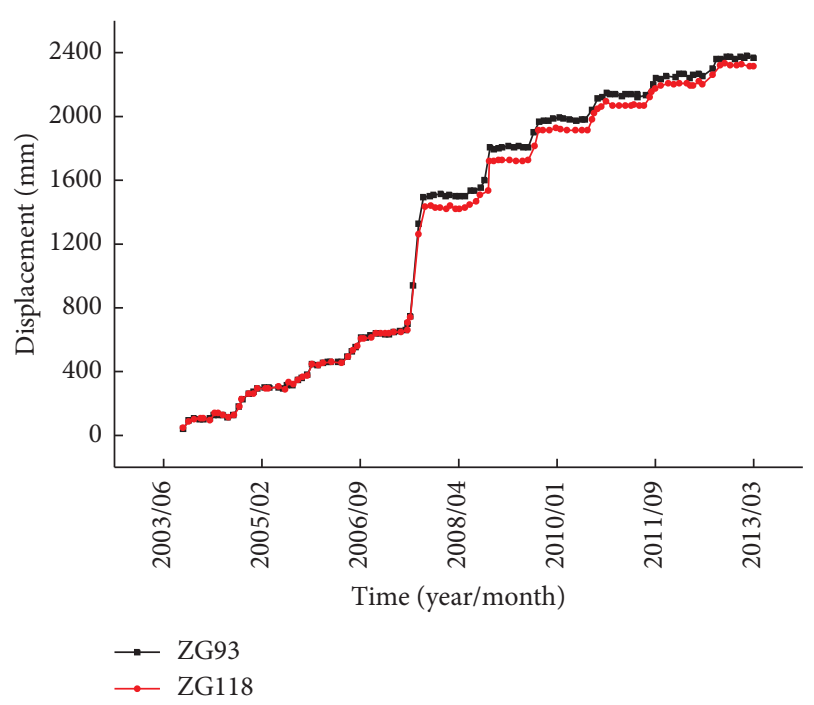

FIGURE 5: Observed displacements along with time in Baishuihe landslide.

Almost all the neural networks used in this paper can fit the trend component curve well, whose R2 are all close to 1 . After the decomposition by the Wavelet Analysis, the trend component curve has S-shaped characteristics, which is smooth and easy to fit. The prediction curves show that the GA-Elman model has better prediction performance compared with other models, whose RMSE and MAPE are relatively low. Compared with the nonoptimized Elman network, the RMSE of the GAElman model decreases by $50 \%$, and the MAPE of the GAElman model decreases by $75 \%$. It shows that the optimization of the Genetic Algorithm has significantly improved the prediction capability of the Elman Neural Network model. The BP Neural Network has relatively large errors due to the uncertainty of the initial weights and the tendency to fall into local minimums. The optimized BP neural model has improved the prediction accuracy.

3.4. Fitting and Prediction of the Periodic Component Displacement. The periodic component displacement is fitted and predicted based on the proposed model. The parameters used in the Genetic Algorithm are consistent with the values in subsection 3.3. A 3-layer Elman network with 20 hidden nodes is constructed. The fitting and prediction curves are shown in Figures 9 and 10, respectively. It can be seen that the periodic component curve is complicated, whose fitting effect is worse than that of the trend term. The R2 is 0.9160 , and the RMSE is 31.3034. However, it can still express the slope displacement time series of the Baishuihe landslide completely. The prediction results show that the RMSE of the monitoring point ZG93 is 4.9447, and the MAPE of the monitoring point ZG93 is 27.0301, which means that the prediction accuracy is high. Then, other neural network models are employed to fit and predict the slope surface deformation. The results are shown in Figures 11 and 12. The evaluation indicators are summarised in Table 2. We can know that the GA-Elman model has the best prediction effect and the highest prediction accuracy. The fitting effects of periodic component displacement through the neural networks are similar. When it comes to the prediction, the RMSE and MAPE through the original Elman model are 50\% higher than those through the optimized Elman model, which indicates that the Genetic Algorithm plays an important role in the prediction. Compared with other networks, Elman and SVM can predict the trend of the periodic component more accurately. The BP network falls into a local minimum value after training, which results in a large deviation of the peak displacement in the prediction curve. In a word, the proposed GA-Elman model has the best prediction capability and optimization performance.

3.5. Prediction of Cumulative Displacement. The cumulative displacement of the slope is the sum of the trend component and the periodic component when the random component is ignored. The fitting displacement is consistent with the monitoring displacement, and the predicted displacement is also close to the monitoring displacement (Figure 13). The R2 of the ZG93 monitoring point is 0.9900 for fitting, and the RMSE is 7.5834 for the prediction. The prediction results based on the GA-Elman model through Wavelet Analysis are better than those through other neural models.

\section{Slope Surface Monitoring Test (Inclination Angle)}

To illustrate the applicability of the proposed GA-Elman model on the prediction of the slope surface deformation, laboratory slope tests are conducted. The length of the model is $1165 \mathrm{~mm}$, the width is $700 \mathrm{~mm}$, and the height is $400 \mathrm{~mm}$ (Figure 14). The base layer and the surface layer are both prepared with the Edosaki sand. The thickness of the surface layer is $100 \mathrm{~mm}$, and the inclination angle is $36^{\circ}$. Five tilt sensors are placed on the surface. The artificial rainfall with a 


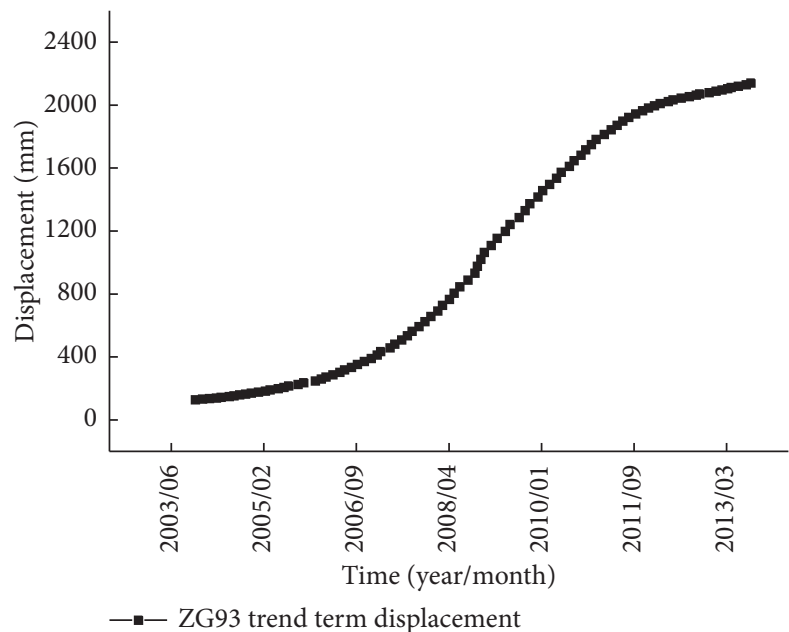

(a)

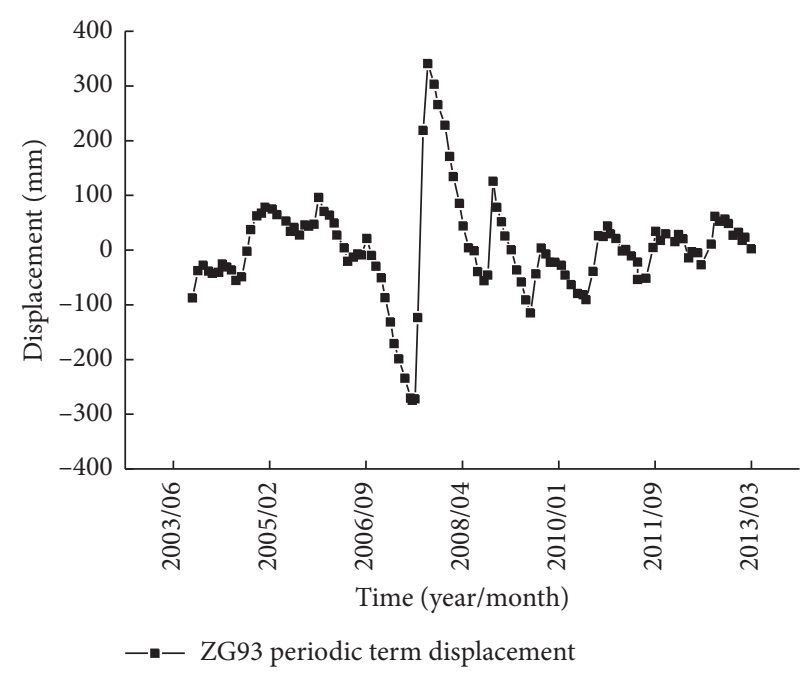

(b)

Figure 6: Results of wavelet analysis: (a) the trend component displacement after decomposition; (b) the periodic component displacement after decomposition.

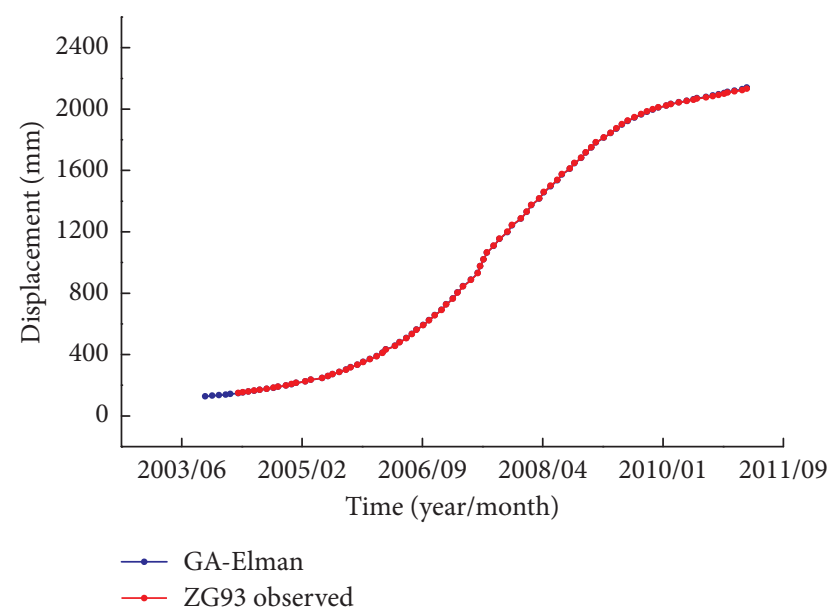

(a)

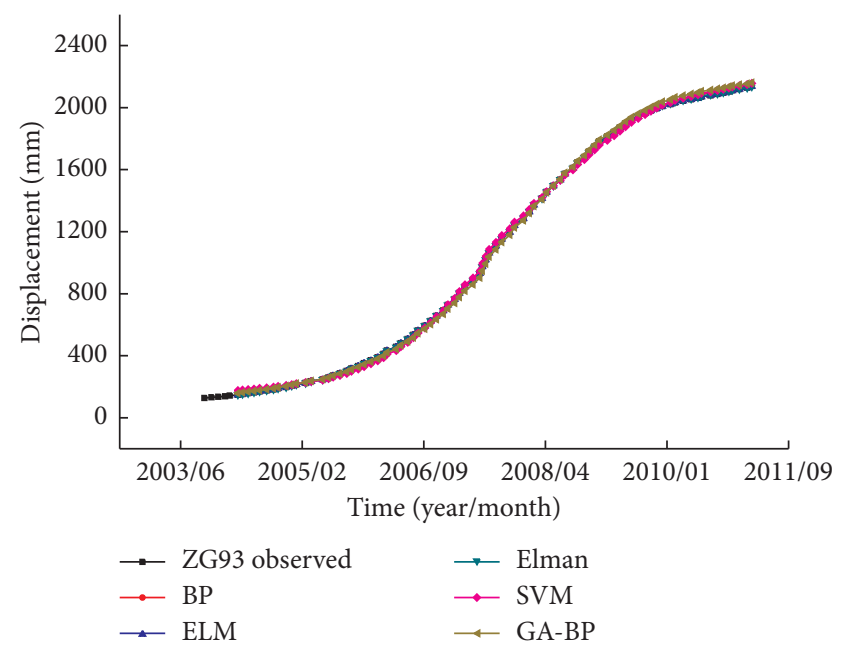

(b)

FIGURE 7: Comparisons between the observed displacements and fitting curves through models for point ZG93 in the Baishuihe landslide: (a) GA-Elman model; (b) other models.

constant intensity of $70 \mathrm{~mm} / \mathrm{h}$ is applied, which can cause the failure of the slope.

During the gradual failure of the slope, the tilt sensor T5 at the bottom of the landslide can monitor the acceleration tilt stage. The T4 and T3 can monitor a similar movement phenomenon. In the prediction of this slope, the data from T5 are used.

The laboratory test can describe the whole development process of the landslide from the slipping to the destruction. Nonexternal factor-induced periodic change exists. Therefore, the decomposition of the time series model is not necessary for the preprocessing. The inclination data can be directly inputted into the optimized GA-Elman model.

In this test, the total time of recorded data for the slope inclination is around 1716s. In the first 1334s, the inclination angle continues changing, which changes from the creep state to the destruction state. Then the inclination angle does not change. To ensure the validity of the measured data, the adjacent inclination angle variations in the selected 1334 sets of data are analyzed. Finally, 245 sets of data are selected for fitting. The data of the first 200 sets are regarded as the training data, during which the inclination data of every continuous $5 \mathrm{~s}$ is set as training input and the inclination data of the 6th second are considered as the expected output. The remaining 45 sets of data can be regarded as the prediction data, which is used to predict the inclination angle of the slope.

The proposed GA-Elman model is used to predict the inclination data from the model tests, where the number of the individuals is set to 60 , the maximum genetic algebra is set to 50 , and other parameters are the same as the above. 




ZG93 observed

$\rightarrow$ GA-Elman



- ZG93 observed

$\rightarrow$ ELM

$\rightarrow$ Elman

(a)

(b)

Figure 8: Prediction performance of different models: (a) GA-Elman model; (b) other models.

TABLE 1: Comparisons of the evaluation indicators on point ZG93 of the trend component displacement prediction models.

\begin{tabular}{|c|c|c|c|c|}
\hline \multirow{3}{*}{ Models } & \multicolumn{4}{|c|}{ ZG93 } \\
\hline & \multicolumn{2}{|c|}{ Fitting data } & \multicolumn{2}{|c|}{ Prediction data } \\
\hline & RMSE & $R^{2}$ & RMSE & MAPE \\
\hline $\mathrm{BP}$ & 6.4716 & 0.9999 & 8.6142 & 0.3254 \\
\hline ELM & 0.1679 & 0.9900 & 3.9101 & 0.1643 \\
\hline Elman & 2.9044 & 0.9900 & 7.9343 & 0.3421 \\
\hline SVM & 11.3004 & 0.9996 & 11.9867 & 7.8607 \\
\hline GA-BP & 18.1316 & 0.9995 & 2.1376 & 0.0735 \\
\hline GA-Elman & 2.2347 & 0.9900 & 2.4907 & 0.0877 \\
\hline
\end{tabular}



FIGURE 9: Fitting performance through GA-Elman model on point ZG93 in the Baishuihe landslide displacement.

The fitting and prediction curves are shown in Figures 15 and 16, which are compared with those obtained from other static neural network algorithms. The evaluation indexes from different models are calculated and summarised in Table 3.
Figure 17 shows that the fitting curves are very similar to all models, which can show the variations of the slope inclination angles during the landslide. Among the prediction curves of the inclination angles, the predicted results from GA-Elman performed the best (Figure 18). The values of 


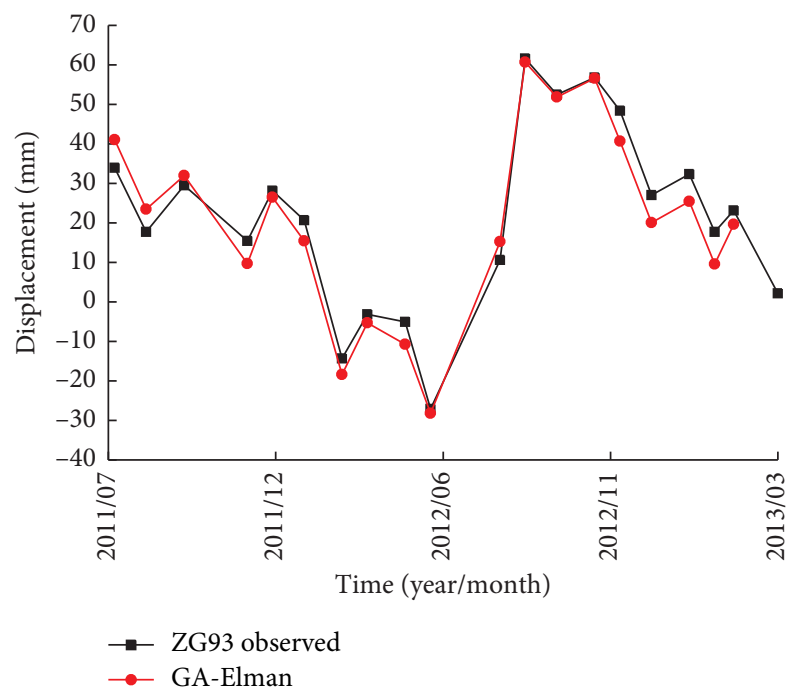

FIGURE 10: Prediction performance through the GA-Elman model on point ZG93 in the Baishuihe landslide displacement.

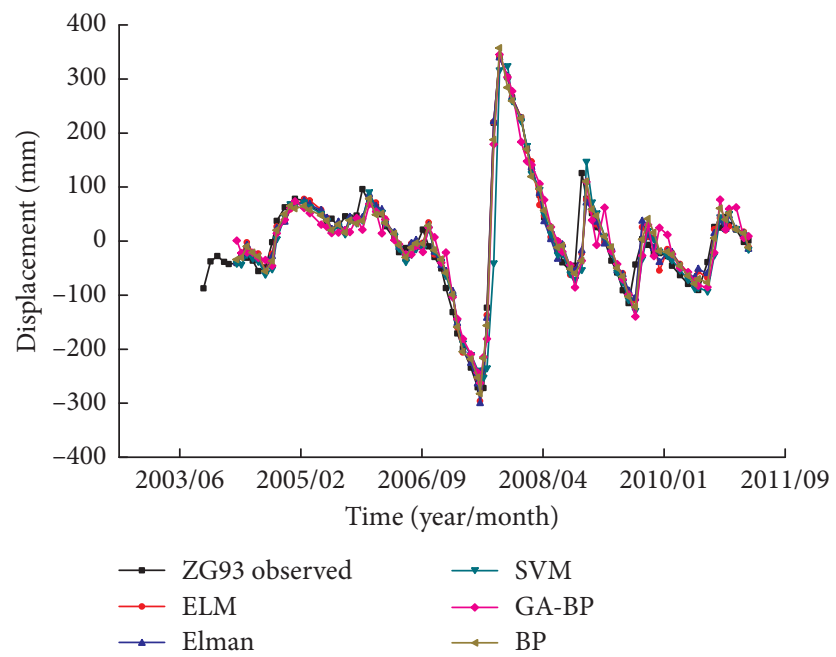

FIGURE 11: Fitting performance through other models on point ZG93 in the Baishuihe landslide displacement.

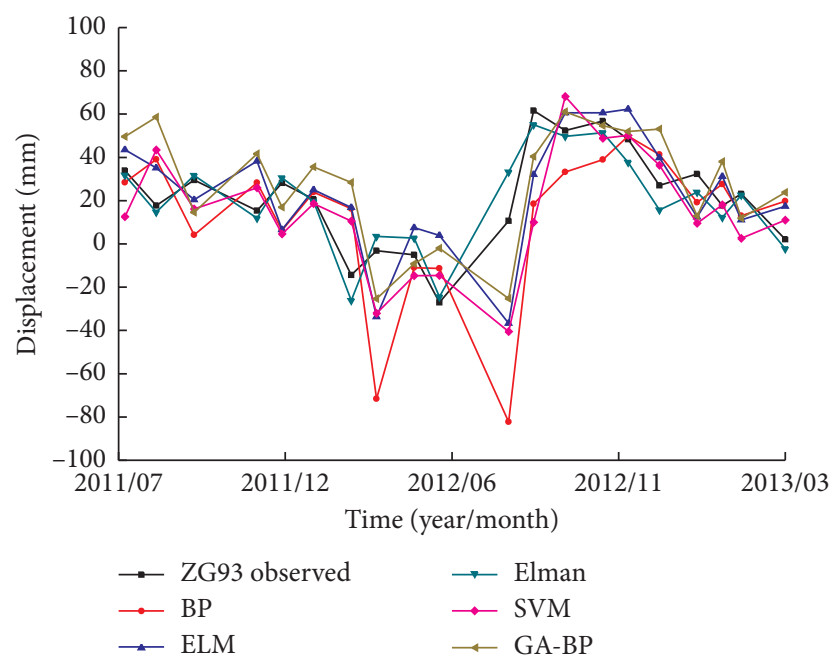

FIGURE 12: Prediction performance through other models on point ZG93 in the Baishuihe landslide displacement. 
TABLE 2: Comparisons of the evaluation indicators on point ZG93 of the periodic trend displacement prediction models.

\begin{tabular}{lcccc}
\hline \multirow{2}{*}{ Models } & \multicolumn{2}{c}{ ZG93 } & \multicolumn{2}{c}{ Prediction data } \\
& RMSE & Fitting data & & RMSE \\
\hline BP & 26.8591 & 0.9346 & 31.4256 & MAPE \\
ELM & 24.0227 & 0.9375 & 21.2309 & 172.2863 \\
Elman & 23.8016 & 0.9680 & 7.8800 & 54.4847 \\
SVM & 16.4935 & 0.8405 & 16.5443 & 148.0590 \\
GA-BP & 25.6514 & 0.8945 & 22.3957 & 175.6390 \\
GA-Elman & 31.3034 & 0.9160 & 4.9447 & 27.0301 \\
\hline
\end{tabular}

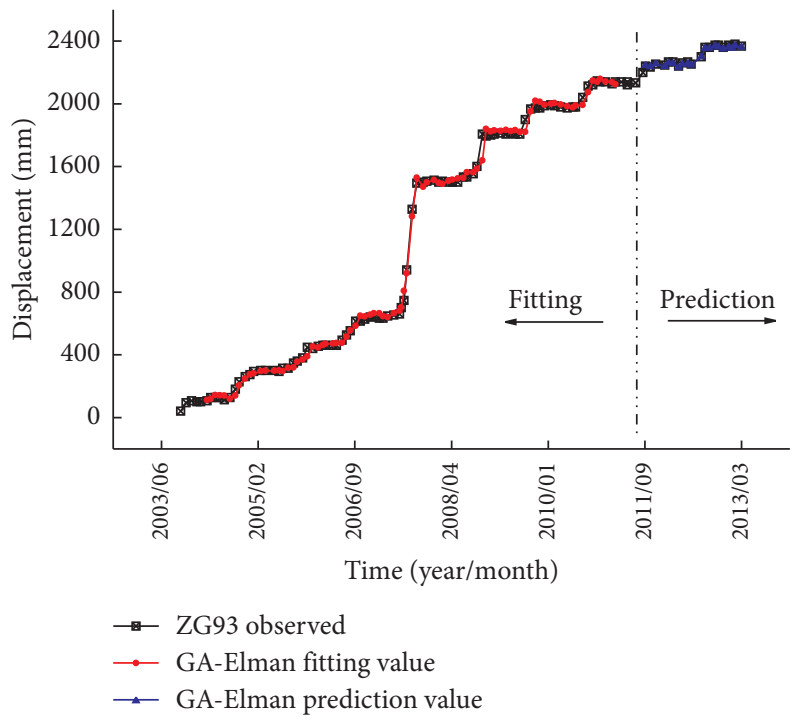

Figure 13: Predicted cumulative displacement through different models for point ZG93 in the Baishuihe landslide.

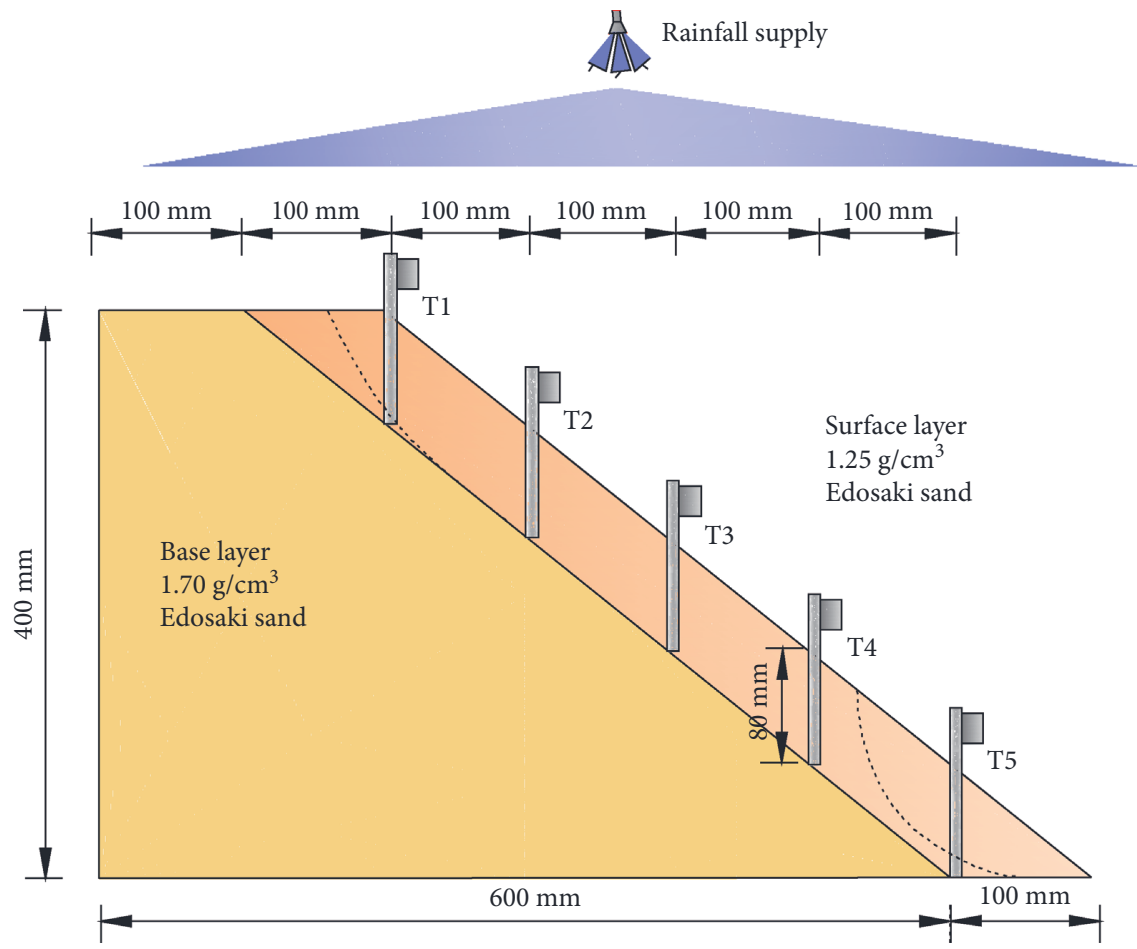

FIGURE 14: The cross-section of the slope model and the setup of apparatuses in the laboratory test. 


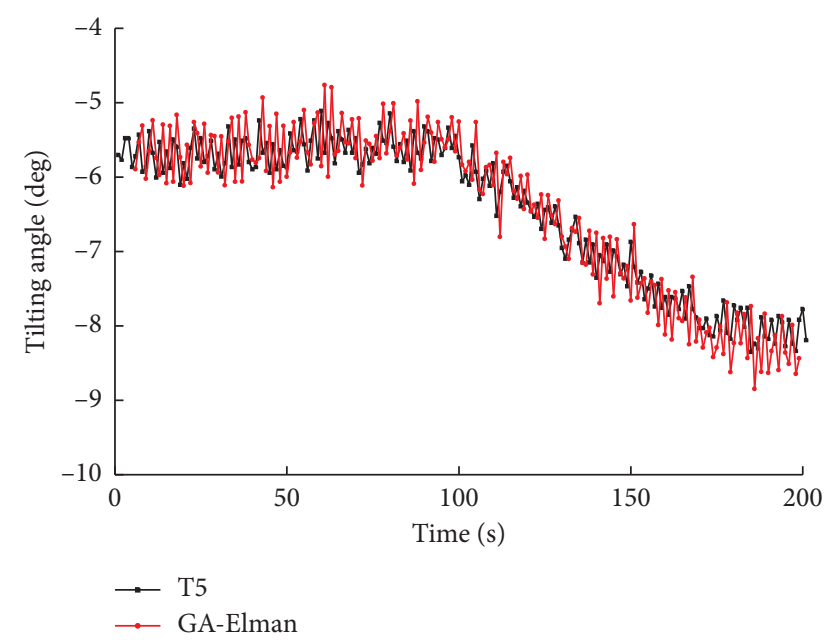

Figure 15: Fitting inclination angles through GA-Elman model.

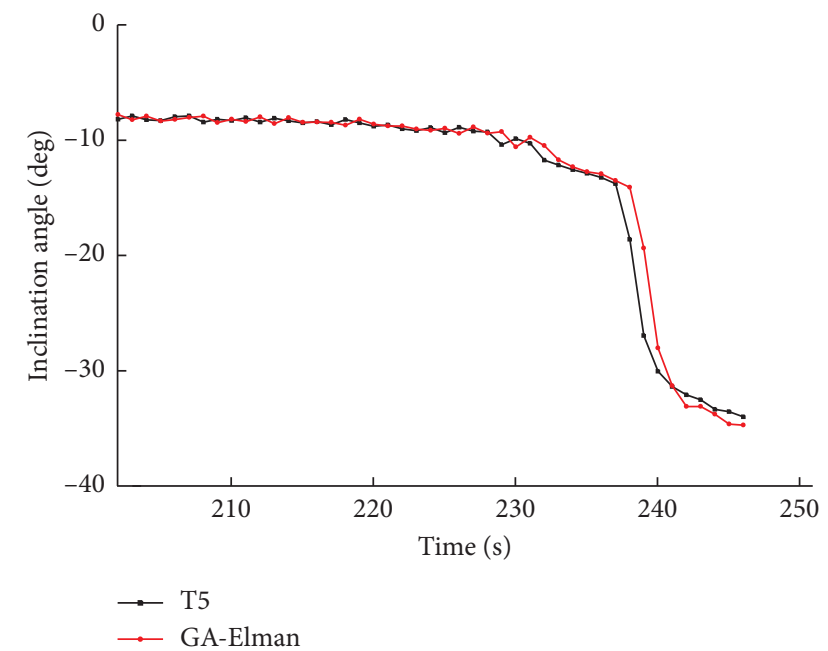

FIGURE 16: Prediction performance of GA-Elman on the inclination angle of $\mathrm{T} 5$.

TABle 3: Comparisons of the evaluation indicators on the inclination angle of $\mathrm{T} 5$ through different models.

\begin{tabular}{lcccc}
\hline \multirow{2}{*}{ Models } & \multicolumn{2}{c}{ T5 } \\
& \multicolumn{2}{c}{ Fitting data } & \multicolumn{2}{c}{ Prediction data } \\
& RMSE & $R^{2}$ & RMSE & MAPE \\
\hline BP & 0.4416 & 0.9914 & 3.6805 & 13.5462 \\
ELM & 0.2967 & 0.9961 & 1.6457 & 8.6666 \\
Elman & 0.3439 & 0.9948 & 1.0416 & 4.6987 \\
SVM & 0.5132 & 0.9888 & 1.0753 & 3.4309 \\
GA-BP & 0.7052 & 0.9801 & 1.4801 & 4.5226 \\
GA-Elman & 0.3097 & 0.8955 & 0.5608 & 3.0822 \\
\hline
\end{tabular}

RMSE and MAPE are 0.5608 and 3.0822, respectively. Compared with other models, the prediction accuracy improved a lot. The optimized Elman Neural Network has better prediction capability than the original Elman Neural Network, which indicates that the determination of initial

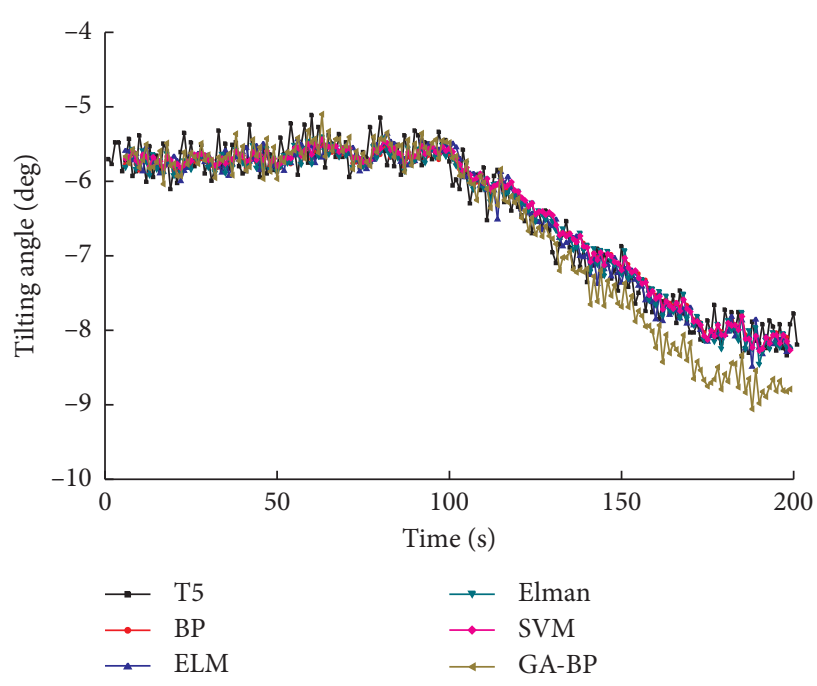

FIGURE 17: Fitting inclination angles through other models.

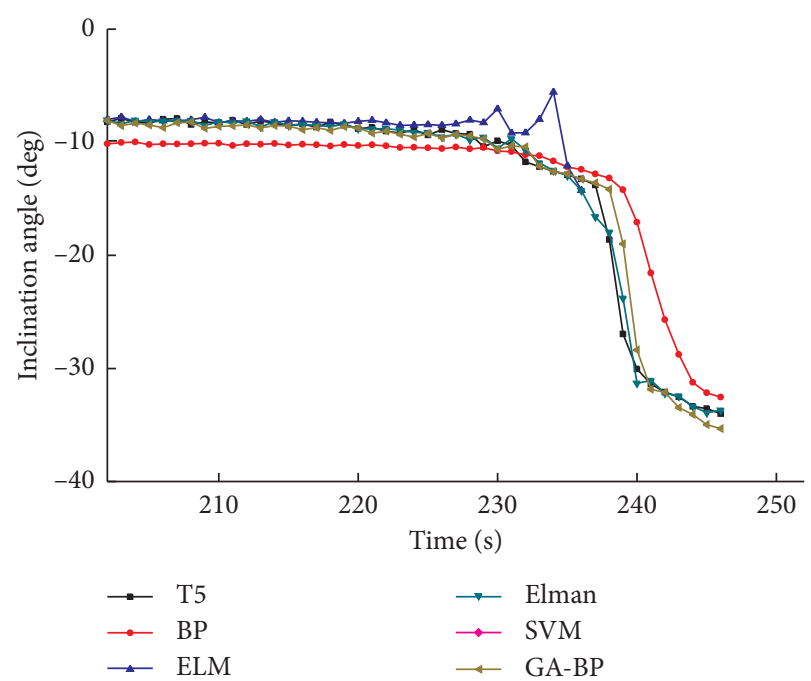

Figure 18: Prediction performance of other models on the inclination angle of $\mathrm{T} 5$.

weight and the threshold play an important role in the prediction. Therefore, the proposed model can predict the inclination angle well.

\section{Conclusions}

The Elman Neural Network optimized by Wavelet Analysis and Genetic Algorithm is proposed, which is employed to predict the slope surface deformation (displacement and inclination angle). The proposed model is validated by the displacement data of the Baishuihe slope and the inclination data of the laboratory tests. The main conclusions are as follows:

(a) The slope surface deformation is affected by both the internal and external factors, which can be decomposed through Wavelet Analysis and the time series 
model. The prediction accuracy can improve a lot. This method can be used to preprocess the slope surface deformation data.

(b) Based on the on-site monitoring displacement data of the Three Gorges area and the inclination angle data of the laboratory tests, the proposed GA-Elman Neural Network model can predict the slope surface displacement and inclination angle with high accuracy.

(c) Compared with the prediction results from other models, the optimized Elman Neural Network has better performance in the prediction of the nonlinear slope surface characteristics. The BP Neural Network, optimized by the Genetic Algorithm, performs better than the traditional BP Neural Network in the prediction.

\section{Data Availability}

The datasets used or analyzed during the current study are available from the corresponding author upon reasonable request.

\section{Conflicts of Interest}

The authors have no conflicts of interest with respect to the research, authorship, and publication of this paper.

\section{Acknowledgments}

This research was funded by Key Research and Development Program of China Railway Corporation (Project 2017G007D).

\section{References}

[1] D. Alexander, "Urban landslides," Progress in Physical Geography: Earth and Environment, vol. 13, no. 2, pp. 157-189, 1989.

[2] T. W. J. V. Asch, J. P. Malet, and L. P. H. V. Beek, “Techniques, issues and advances in numerical modelling of landslide hazard," Bulletin De La Societe Geologique De France, vol. 178, no. 2, pp. 65-88, 2007.

[3] S. H. Beville, B. B. Mirus, B. A. Ebel, G. G. Mader, and K. Loague, "Using simulated hydrologic response to revisit the 1973 Lerida Court landslide," Environmental Earth Sciences, vol. 61, no. 6, pp. 1249-1257, 2010.

[4] J. Du, K. Yin, and S. Lacasse, "Displacement prediction in colluvial landslides, three gorges reservoir, China," Landslides, vol. 10, no. 2, pp. 203-218, 2013.

[5] Y. Yin, H. Wang, Y. Gao, and X. Li, "Real-time monitoring and early warning of landslides at relocated wushan town, the three gorges reservoir, China," Landslides, vol. 2, no. 7, pp. 339-349, 2010.

[6] A. Federico, M. Popescu, G. Elia, C. Fidelibus, G. Internò, and A. Murianni, "Prediction of time to slope failure: a general framework," Environmental Earth Sciences, vol. 66, no. 1, pp. 245-256, 2012.

[7] W. D. Wang, X. Qu, and P. Liu, "Prediction on landslide displacement using a combination model with optimal weight," Natural Hazards, vol. 96, pp. 1121-1139, 2019.
[8] X. Li, J. Kong, and Z. Wang, "Landslide displacement prediction based on combining method with optimal weight," Natural Hazards, vol. 61, no. 2, pp. 635-646, 2012.

[9] M. Saito, "Forecasting the time of occurrence of a slope failure," in Proceedings of the 6th International Conference on Soil Mechanics and Foundation Engineering, no. 2, pp. 537541, Montreal, Canada, September 1965.

[10] S. Hayashi, F. Komamura, and B. Park, "On the forecast of time to failure of slope-approximate forecast in the early period of the tertiary creep," Landslide, vol. 25, 1988.

[11] A. Federico, M. Popescu, C. Fidelibus, and G. Internò, "On the prediction of the time of occurrence of a slope failure: a review," Landslides: Evaluation and Stabilization/Glissement de Terrain: Evaluation et Stabilisation, vol. 2, no. 2, pp. 979-983, 2004.

[12] K. Yin and T. Yan, "Landslide prediction and relevant models," Chinese Journal of Rock Mechanics and Engineering, vol. 1, pp. 1-8, 1996.

[13] L. Z. Wu, S. H. Li, R. Q. Huang, and Q. Xu, "A new grey prediction model and its application to predicting landslide displacement," Applied Soft Computing, vol. 95, no. 95, Article ID $106543,2020$.

[14] J. L. Xu and H. Ma, "Discussion on prediction methods of landslide imminent sliding," Chinese Journal of Geological Hazard and Control, vol. 9, pp. 364-369, 1998.

[15] J. Du, K. L. Yin, and B. Chai, "Study of displacement prediction model of landslide based on response analysis of inducing factors," Chinese Journal of Rock Mechanics and Engineering, vol. 28, no. 9, pp. 1783-1789, 2009.

[16] J. Zhang, K. L. Yin, and J. J. Wang, "Displacement prediction of baishuihe landslide based on time series and PSO-SVR model," Chinese Journal of Rock Mechanics and Engineering, vol. 34, no. 2, pp. 382-391, 2015.

[17] Z. Liu, J. Shao, W. Xu, H. Chen, and C. Shi, "Comparison on landslide nonlinear displacement analysis and prediction with computational intelligence approaches," Landslides, vol. 11, no. 5, pp. 889-896, 2014.

[18] J. Xie, T. Uchimura, and P. Chen, "Relationship between displacement and tilting angle of the slope surface in shallow landslides," Landslides, vol. 16, pp. 1243-1251, 2019.

[19] J. Xie, T. Uchimura, G. Wang et al., "Predicting the sliding behavior of rotational landslides based on the tilting measurement of the slope surface," Engineering Geology, vol. 269, no. 269, Article ID 105554, 2020.

[20] Q. Xu, M. Tang, K. Xu, and X. Huang, "Research on spacetime evolution laws and early warning-prediction of landslides," Chinese Journal of Rock Mechanics and Engineering, vol. 27, no. 6, pp. 1104-1112, 2008.

[21] G.-B. Huang, D. H. Wang, and Y. Lan, "Extreme learning machines: a survey," International Journal of Machine Learning and Cybernetics, vol. 2, no. 2, pp. 107-122, 2011.

[22] L. Ebadi, H. Z. M. Shafri, and S. B. Mansor, "A review of applying second-generation wavelets for noise removal from remote sensing data," Environmental Earth Sciences, vol. 70, pp. 2679-2690, 2013.

[23] F. Huang, K. Yin, and G. Zhang, "Landslide displacement prediction using discrete wavelet transform and extreme learning machine based on chaos theory," Environmental Earth Science, vol. 75, no. 20, pp. 1376-1393, 2016.

[24] I. Daubechies, Ten Lectures on Wavelets, Mathematics of Computation, Philadelphia, Pennsylvania, 1993.

[25] Q. Xu and X. B. Huang, Handbook of Early Warning and Forecast of Landslide Hazards in the Three Gorges Reservoir Area, Geological Publishing House, Beijing, China, 2014. 
[26] Q. XU, "Theoretical studies on prediction of landslides using slope deformation process data," Journal of Engineering Geology, vol. 20, no. 02, pp. 145-151, 2012.

[27] S. q. LU, Q. l. YI, and W. YI, "Study on dynamic deformation mechanism of landslide in Drawdown of reservoir water level-baishuihe Landslide in three gorges reservoir area," Journal of Engineering Geology, vol. 22, no. 5, pp. 869-875, 2014.

[28] Y. Bai, H. Xu, and H. Ling, "Drought-flood variation and its correlation with runoff in three headstreams of Tarim River, Xinjiang, China," Environmental Earth Sciences, vol. 71, no. 3, pp. 1297-1309, 2014.

[29] J. Grefenstette, "Optimization of control parameters for genetic algorithms," IEEE Transactions on Systems, Man, and Cybernetics, vol. 16, no. 1, pp. 122-128, 1986.

[30] F. Ren, X. Wu, K. Zhang, and R. Niu, "Application of Wavelet Analysis and a particle swarm-optimized support vector machine to predict the displacement of the shuping landslide in the three gorges, China," Environmental Earth Sciences, vol. 73, no. 8, 2014.

[31] Y. Cao, K. Yin, D. E. Alexander, and C. Zhou, "Using an extreme learning machine to predict the displacement of steplike landslides in relation to controlling factors," Landslides, vol. 13, no. 4, pp. 725-736, 2016. 